\title{
An unusual foreign body in the paranasal sinuses
}

\author{
Rajnish Sharma · R. Minhas · N. Mohindroo
}

\begin{abstract}
We report a case of 38-year-old male who was reffered from eye OPD with a retained foreign body in the periobita of Rt eye extending into the Rt ethmoid and sphenoid sinuses after six months of injury. The foreign body was removed through external ethmoidectomy and incision on the periorbita of Rt eye.
\end{abstract}

Keywords Foreign body - Rt. Periorbita - Ethmoid and sphenoid Sinuses

\section{Introduction}

The foreign body in the paranasal sinuses is extremely rare particularly in frontal, ethmoid sinuses in comparison to maxillary \& sphenoid sinuses. These foreign bodies are mainly traumatic in origin (maxillofacial) which occur directly as a result of external trauma to the respective sinsus or indirectly through the orbital or palatal injuries. There can be visual morbidity and functional residual deficit when the route of the these injuries is orbital. If these foreign bodies are radio opaque, then these can be easily recognisable by X-Ray. However to know the exact extent of damage caused by these foreign bodies, can be made out by CT-

R. Sharma ${ }^{1}$ • R. Minhas ${ }^{2}$ • N. Mohindroo ${ }^{3}$

${ }^{1}$ Post Graduate Student

${ }^{2}$ Registrar

${ }^{3}$ Associate Professor

Department of ENT,

I.G. Medical College

Shimla, India

R. Sharma $(\bowtie)$

C/o Ganpati Bhawan

2nd Floor, Aira Holmes,

Near Happy Child School

Chotta Shimla,

Shimla (H.P.) - 171 002, India

e-mail: rajnishvasu@gmail.com
Scan only. If the foreign bodies are large enough then their removal is not easy by routine Endoscopy. The rhinosurgical approaches also looks to be inadequate keeping in view the operative and post operative morbidity as the important structure like optic nerve, carotid artery, cribriform plate and orbit itself lies in close relation to these sinuses. If foreign body is left behind, it will cause a foreign body granuloma and there will be local symptoms like local irritation in the eye, lacrimation, epistaxis, discharge from the nose and some time foreign body sensations at the particular site.

\section{Case report}

A 38-year-old male sustained injury in Rt eye while he was cutting wood six months back. He was operated at district hospital where small bits of wood from medial side of the orbit (sclera) were removed and stitches applied on the wound near lower eyelid and discharged on antibiotic and analgesic. But the main complaint of patient like pain and swelling, lacrimation from right eye continued and later patient was referred to IGMC Shimla, Ophthalmology department where vision was 6/36 Rt eye and ocular movement were restricted on lateral side. CT Scan was done and medical rectus haematoma was suspected and discharged on systemic steroid, antibiotic and analgesics.

After two weeks of intensive therapy, patient again reviewed in eye OPD with the same complaints as earlier.

Vision in Rt eye was 6/24, with restricted ocular movements laterally.

Again plain and contrast CT scan of orbit and head was done which showed soft tissue swelling in the region of medical rectus and a linear opacity measuring $2.75 \mathrm{~cm}$. in length which was hyerdense on Rt side medially. Along the track lamina papyracea was dehiscent (Fig. 2). This opacity was extending into ethmoid sinus Rt and sphenoid sinus Rt.

This CT scan findings were suggestive of foreign body in the region of medial rectus going into ethmoid sinus with foreign body granuloma (Fig. 1) and patient was shifted 
to otorhino-laryngology and head and neck department for further management. The patient was investigated for routine blood and urine and kept for endoscopic removal of foreign body.

The endoscopic examination was done under local anaesthesia and only part of foreign body was visualised and removal could not be possible. Immediately a decision to undertake external enthmoidectomy approach for the removal of foreign body was undertaken. The lamina papyracea was dehiscent through which foreign body was seen and it was extending into lower deep structures. But anterior end was not visualized as it was embedded in the periorbita tissue. A incision was given on the periorbita and this foreign body was removed through this unusual route (Fig. 3). The operative haemostasis was attained and posterior wall of sphenoid examined which was found to be normal but small destruction of anterior wall of sphenoid was present. The post operative eye consultation was done and vision of Rt eye was 6/24 and fundus was normal. Ocular movement

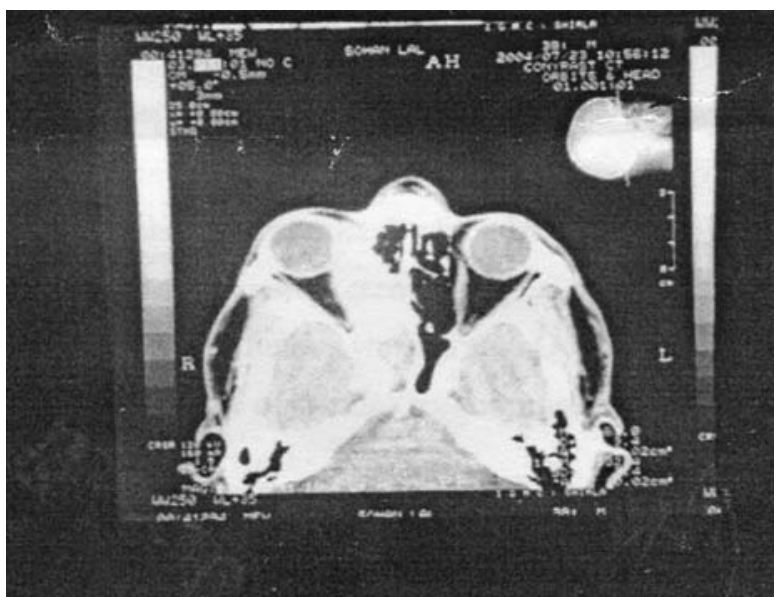

Fig. 1 CT Scan Showing FB Rt ethmoid Sinus

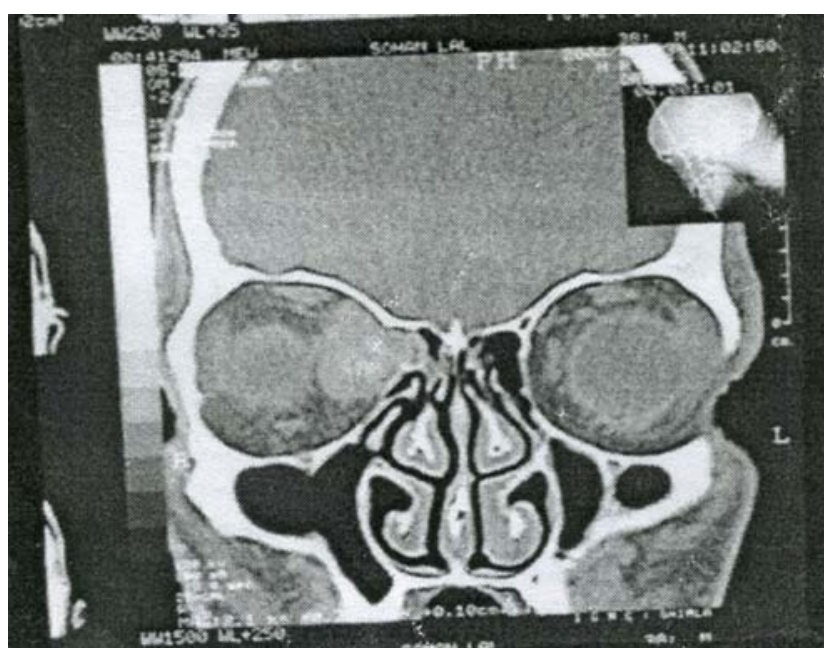

Fig. 2 FB granuloma in Rt eye and lamina Papyracea eroded were restricted laterally and on CT Scan- entrapment of medial rectus muscle was suggested and medial rectus release was considered after 6-8 weeks.

After 6-8 weeks - again CT scan was done in which patient showed improvement. There was thickening of medial rectus. But vision of the patient was $6 / 18$ with ocular movements returning to normal. (Fig. 4)

\section{Discussion}

Foreign body in the ethmoid \& sphenoid sinuses are mostly associated with orbital trauma with retention of foreign body and local symptom. These foreign bodies which are embedded in the orbit, ethmoid and sphenoid sinuses do not cause much symptoms except local irritation, pain on movement of eye and sometimes nasal discharge. The clinical diagnosis is not easy if the patient presents after sometime of injury. The X-Ray is also not very useful except to

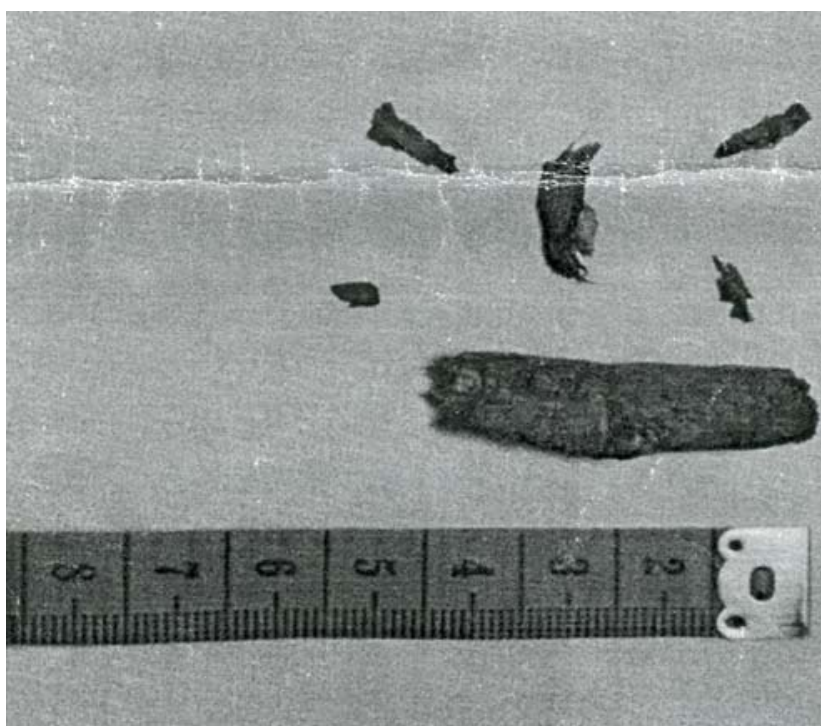

Fig. 3 FB removed from the above mentioned area of Rt eye

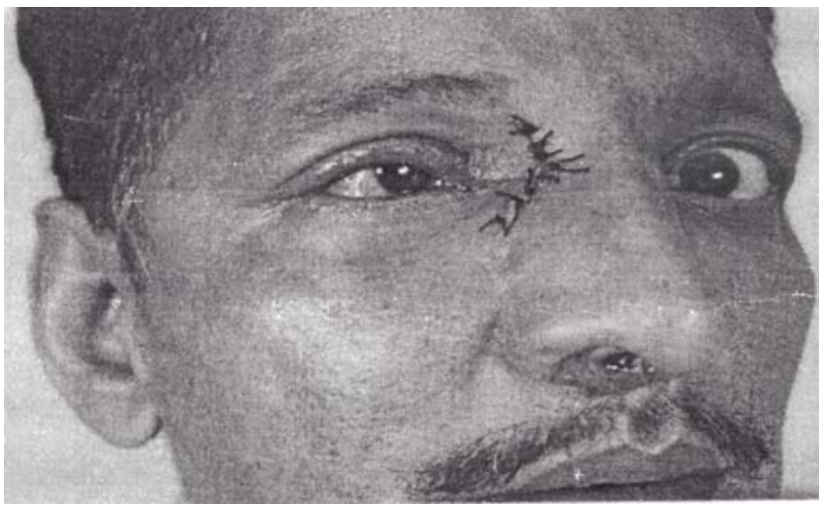

Fig. 4 Showing recovery in Ocular movement 
know the presence of foreign body if it is radio opaque. But $\mathrm{CT}$ scan is very useful to know the size, extent and relation of the foreign body to underlying structures.

Although metallic foreign bodies have been described in literature by Hirschberg and by steindroff and also by Himadri Datta, Krishnendu Sarkar et al (2001), but this type of wooden piece could not be found in any literature or journal available with us. As this patient with retained foreign body presented after 6 months with only symptoms of pain, foreign body sensation in eye, lacrimation, diminution of vision and movement of eye affected, the removal of such big foreign body was not easy by endoscopic approach and also by external ethmoidectomy alone and both procedure in-combination were found to be effective without morbidity.

\section{Reference}

1. Payne RF (1967) FB in frontal sinus. British J of Radiology 40:778-780

2. Etheringtion RJ, Hourihan MD (1989) Localisation of intraocular and intraorbital FB using CT. Clin Radiol 40:610-615

3. Ranko Mladina (1992) Endoscopic sinsus surgery: A metallic foreign bodyat sphenoethmoid junction. The journal of laryngology and otology, vol. 106, pp 998-999

4. Agarwal PK, Kumar H, Srivastava PK (1993) Unusual orbital foreign bodies. Indian J Ophthalmol 41:125-127

5. Himadri Datta et al (2001) A unusual case of retained metallic arrow head in the orbit and sphenoid sinus. Indian J ophthalmol 49:197-198

6. Vinita Singh et al (2004) An unusual Intraorbital Foreign body. Indian J ophthalmol 52:64-65 